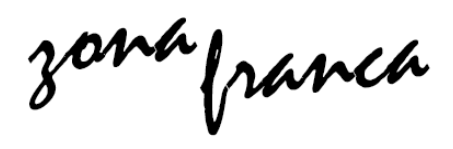

\title{
Cuestiones de género en una ciudad media. Consideraciones en torno de políticas públicas
}

\author{
Patricia Pérez * María Eugenia Iturralde **
}

\section{Resumen}

El propósito general de nuestro trabajo pretende contribuir al conocimiento de especificidades en los procesos institucionales vinculados a problemáticas de género que se dan en Olavarría. Cuando mencionamos la idea de procesos institucionales, nos referimos en principio a una suma de actividades que interactúan y se articulan para incidir en un conjunto poblacional determinado; pero también hacemos mención a los modos -que tienen las instituciones relacionadas con el tratamiento de la violencia de género- mediante los que se pueden regular e incluso controlar los comportamientos de las personas. Esas instituciones sociales son las que implementan políticas públicas en el orden local. Si bien los problemas de género que nos afectan no han de reducirse a un entendimiento asociado únicamente con la existencia de la violencia física, lo cierto es que este recorte particular es el que más se haya representado en los incumplimientos institucionales del Estado argentino en temas de Derechos Humanos. En este sentido, se verifica también la aparición de un dispositivo para atención de la violencia que surge como correlato de la demanda, desde el Estado municipal.

\footnotetext{
* Producciones e investigaciones comunicacionales y sociales de la ciudad intermedia- Estudios de Comunicación, Cultura y Género- Facultad de Ciencias Sociales- Universidad Nacional del centro de la Provincia de Buenos Aires. Contacto: pataperez@gmail.com

** Producciones e investigaciones comunicacionales y sociales de la ciudad intermedia- Estudios de Comunicación, Cultura y Género- Facultad de Ciencias Sociales - Universidad Nacional del centro de la Provincia de Buenos Aires- Consejo Nacional de Investigaciones Científicas y Técnicas. Contacto: eugeniaiturralde@gmail.com
}

Pérez, Patricia; Iturralde, María Eugenia. "Cuestiones de género en una ciudad media. Consideraciones en torno de políticas públicas" en Zona Franca. Revista del Centro de estudios Interdisciplinario sobre las Mujeres, y de la Maestría poder y sociedad desde la problemática de Género, N²7, 2019 pp.171-196. ISSN, 2545-6504 Recibido: 30 de junio 2019; Aceptado: 12 de noviembre 2019

Revista Zona Franca- Centro de estudios interdisciplinario sobre las mujeres (CEIM)- Maestría poder y sociedad desde la problemática de género (MG), Rosario, Argentina. ISSN, 2545-6504 http://zonafranca.unr.edu.ar/index.php/ZonaFrancal Numero 27 (2019). 


\begin{abstract}
Palabras claves: Políticas públicas- Género- Ciudad mediaInstitucionalidad
\end{abstract}

Gender issues in a middle range city. Considerations around public policy

\begin{abstract}
The general purpose of this work aims to contribute to the knowledge of specificities in the institutional processes linked to gender issues that occur in Olavarría. When we mention the idea of institutional processes, we refer in principle to a sum of activities that interact and articulate to influence a specific population set; but we also mention the ways -which institutions have to do with the treatment of gender violence- through which the behaviors of people can be regulated and even controlled. These social institutions are those that implement public policies in the local order. Although the gender problems that affect us should not be reduced to an understanding associated only with the existence of physical violence, the truth is that this particular cut is the one that is most represented in the institutional failures of the Argentine State in matters of Human rights. In this sense, it also verifies the appearance of a device for attention of violence that arises as a correlate of the demand, from the municipal State.
\end{abstract}

Keywords: Public policies- Gender- Middle range city Institutionality

\title{
1. Introducción
}

Este trabajo presenta un recorrido por tres aristas: procesos institucionales, ciudades medias y políticas públicas para abordar la violencia de género. Las mismas no son necesariamente rectas sino más bien sinuosas y confluyen básicamente en lo que denominamos atención de las problemáticas de género en la ciudad de Olavarría, específicamente, a partir de lo que hemos denominado procesos institucionales.

Proponemos desmenuzar las especificidades de las aristas a sabiendas que, necesariamente, se entrecruzan en lo concreto; en este sentido, los procesos institucionales adquieren particularidad cuando se desarrollan en lo que

Revista Zona Franca- Centro de estudios interdisciplinario sobre las mujeres (CEIM)- Maestría poder y sociedad desde la problemática de género (MG), Rosario, Argentina. ISSN, 2545-6504 http://zonafranca.unr.edu.ar/index.php/ZonaFranca| Numero 27 (2019). 
denominamos ciudades medias, donde las relaciones formales entre las instituciones se dan en un marco en el que no está exenta -en algunos aspectosla informalidad que revisten ciertas prácticas locales.

Este conjunto de prácticas -tanto formales como informales- las registramos en el contexto de lo que se denomina dispositivo para atención de la violencia, que surge como correlato de la demanda social efectuada desde el feminismo local y operativizada institucionalmente por el Estado municipal. De acuerdo al objeto de estudio, optamos por una estrategia metodológica de carácter cualitativo. Atendimos a la estrategia general de producción de datos -es decir, a procedimientos y métodos para la obtención de información empírica- articulando diferentes técnicas y procedimientos para la recolección de información: entrevistas abiertas, observación participante, elaboración de registros en las reuniones de la Mesa Local (de diciembre de 2015 a diciembre de 2018) y análisis de documentos (corpus legales de la provincia de Buenos Aires, nacionales e internacionales; documentos de la Dirección de Políticas de Género de Olavarría y del Ejecutivo provincial; actas de la Mesa Local contra la Violencia Familiar y de Género de Olavarría). Estas técnicas de producción de información empírica dieron lugar a la interacción y articulación entre datos primarios y datos secundarios.

El objetivo de este trabajo es dar cuenta de cómo se desarrollaron las políticas públicas en la ciudad de Olavarría, en tanto ciudad media, en el marco del surgimiento y consolidación de la Dirección de Políticas de Género (período 2015-2018).

A continuación, desarrollaremos suscintamente las aristas antes mencionadas para otorgar claridad a los aspectos que pretendemos conjugar.

\section{Los espesores de las aristas}

\subsection{Las ciudades medias}

Revista Zona Franca- Centro de estudios interdisciplinario sobre las mujeres (CEIM)- Maestría poder y sociedad desde la problemática de género (MG), Rosario, Argentina. ISSN, 2545-6504 http://zonafranca.unr.edu.ar/index.php/ZonaFrancal Numero 27 (2019). 
Olavarría, puede ser caracterizada como una "ciudad media"; es ciudad homónima del Partido, ubicado en el centro geográfico de la provincia de Buenos Aires tiene -según el último censo poblacional- 111.708 habitantes', en su mayoría ubicados en el casco urbano.

En principio, parece relevante definir la idea de "ciudad media" o de "rango medio" de modo de dejar asentado el territorio desde el que se piensan y analizan estas intersecciones particulares. En este sentido, para la antropóloga Silvia Boggi (2008) -y para categorías analíticas de las Ciencias Sociales- Olavarría puede ser definida como "ciudad media". La autora sostiene que esa categoría se dirime relacionalmente. Su trabajo, crítico con los abordajes más clásicos (cantidad de habitantes, dimensiones espaciales, cualificación de los servicios urbanos con los que se cuenta) propone una no estandarización conceptual de lo que sería la "ciudad media"; más bien, desde los cuestionamientos a las disciplinas tradicionales que se encargaron de las ciudades como la geografía, pone al descubierto la tensión existente desde la lógica antropológica del interjuego ciudad-pueblo" que se da en estas dimensiones espaciales y entramados sociales. También, afirma que no puede pensarse a las ciudades medias "como meros espacios físicos y como variables independientes de la estructura socioeconómica y del sistema capitalista" (2008:13). Nuestro trabajo se inscribe y reflexiona en el marco de las particularidades dadas por las lógicas de vinculación personal, interinstitucional y social propias de estas ciudades medias.

Asimismo, resulta interesante considerar la aproximación que produce Boggi en colaboración con Galván, puesto que sostienen que "las reflexiones en ámbitos académicos y/o profesionales, (...) concentran debates sobre los fenómenos que acontecen en las grandes ciudades, dejando a un lado la posibilidad de construir a

I INDEC, Cuadro P2-D. Provincia de Buenos Aires, partido Olavarría. Población total por sexo e índice de masculinidad, según edad en años simples y grupos quinquenales de edad. Año 2010.

"El Censo poblacional realizado en 2010 indica que en la Provincia de Buenos Aires existen 45 ciudades que tienen una población que oscila entre los 50.000 y los 500.000 habitantes, y 83 que poseen menos de 50.000 (Iturralde, 2017).

Revista Zona Franca- Centro de estudios interdisciplinario sobre las mujeres (CEIM)- Maestría poder y sociedad desde la problemática de género (MG), Rosario, Argentina. ISSN, 2545-6504 http://zonafranca.unr.edu.ar/index.php/ZonaFrancal Numero 27 (2019). 
las ciudades medias y pequeñas como objeto de análisis" (Boggi y Galván, 2016:33). En este sentido, el presente trabajo también se propone aportar conocimientos propios del territorio y para el territorio.

Es posible ligar posiciones con los postulados vertidos por Donna Haraway (1995); se trata de estar a "favor de políticas y de epistemologías de la localización, del posicionamiento y de la situación, en las que la parcialidad y no la universalidad es la condición para que sean oídas las pretensiones de lograr un conocimiento racional. Se trata de pretensiones sobre la vida de la gente, de la visión desde un cuerpo, siempre un cuerpo complejo, contradictorio, estructurante y estructurado, contra la visión desde arriba (...)" (Haraway, 1995:335). La autora explicita: "Yo quisiera una doctrina de la objetividad encarnada que acomode proyectos de ciencia feminista paradójicos y críticos: la objetividad feminista significa, sencillamente, conocimientos situados" (1995:324).

Así, la elaboración de una comprensión exhaustiva sobre algunos aspectos locales resulta también un aporte a nuevos planteos epistemológicos, a partir de significaciones que emanan de cuestiones institucionales vinculadas a políticas públicas de género en ciudades medias.

\subsection{Los procesos institucionales: interinstitucionalidad e intersectorialidad}

Si bien sabemos que los problemas de género no han de acotarse a un entendimiento restringido y asociado únicamente con la existencia de la violencia machista -con preeminencia en el marco de las visibilidades de la violencia física-, lo cierto es que este recorte particular es el que más se halla representado en los incumplimientos institucionales del Estado argentino (constitucionales y sobre todo convencionales) en temas de Derechos Humanos. Tal vez sea porque la violencia física contenga en sí misma las marcas más evidentes (no solamente corpóreas) de esas inobservancias. En este sentido, compartimos con algunas estudiosas

Revista Zona Franca- Centro de estudios interdisciplinario sobre las mujeres (CEIM)- Maestría poder y sociedad desde la problemática de género (MG), Rosario, Argentina. ISSN, 2545-6504 http://zonafranca.unr.edu.ar/index.php/ZonaFranca| Numero 27 (2019). 
latinoamericanas que: "La violencia doméstica es tal vez la temática de género que mayor facilidad de reconocimiento y de incorporación ha tenido dentro de la agenda municipal. Esta apertura se debe a las luchas, trabajos y estudios de las mujeres que, desde la década de 1980, han conseguido hacer públicamente visible la violencia que afecta principalmente a las mujeres en la esfera de la vida privada. Es un problema de género que, al haber adquirido el rango de interés público y de problema de salud pública, motiva la intervención de los gobiernos municipales". (Barrera Bassols y Massolo, 1998:45)

La necesidad de modificar algún aspecto de la realidad circundante -en este caso la violencia física ejercida por cuestiones de género- da lugar a la conformación de una agenda social problemática, en la que se ven involucrados tres tipos de actores: instituciones estatales, proveedores del mercado y organizaciones de la sociedad civil. Cuando un asunto es problematizado se transforma en una cuestión social, que puede ser tomada por el Estado o por la sociedad civil (Oszlak y O'Donnell, 1995). Si las agencias del Estado dan lugar a esa cuestión, ingresa a la agenda gubernamental y las autoridades buscarán una resolución (Ingaramo, 2013).

En el período de análisis, 2015-2018, observamos que el Estado municipal de Olavarría ingresa el abordaje de la violencia física como tema a la agenda gubernamental. En ese sentido, surgen nuevos procesos institucionales que llevaron a las dependencias del estado local a profundizar algunas líneas de acción iniciadas previamente, como por ejemplo sancionar una ordenanza y crear la Dirección de Políticas de Género (DPG), entre otras (Pérez e Iturralde, 2018). Cuando mencionamos la idea de procesos institucionales nos referimos, por un lado, a una suma de actividades que interactúan y se relacionan para incidir en un conjunto poblacional determinado; y por otro a los modos, dispositivos y estructuras -mediante los que se pueden regular e incluso controlar los procederes de las personas- que tienen las instituciones sociales dedicadas al tratamiento de la violencia de género.

Revista Zona Franca- Centro de estudios interdisciplinario sobre las mujeres (CEIM)- Maestría poder y sociedad desde la problemática de género (MG), Rosario, Argentina. ISSN, 2545-6504 http://zonafranca.unr.edu.ar/index.php/ZonaFranca| Numero 27 (2019). 
Situarnos en una ciudad media nos permite conocer tensiones, intenciones, circuitos e intereses que se dan en los entramados propios de la conformación e implementación de las políticas locales destinadas a la atención de las problemáticas de género, específicamente las relacionadas con el tratamiento de la violencia. Retomando las conceptualizaciones, ya clásicas, de Lasswell (2013), este trabajo se propone abordar la comprensión del proceso de las políticas, cuya finalidad es describir y analizar cómo se da el proceso de diseño, implementación, y evaluación.

En este sentido, planteamos abordar diferentes dimensiones, siempre teniendo presente el contexto sociopolítico y económico en el que se inscribe el abordaje de la violencia de género: concurrencia de actores/as disímiles en el territorio, marcos normativos que regulan cómo se debe actuar ante la problemática y dispositivos de atención. Aunque, como ya consignamos es necesario considerar que, en lo concreto, se muestran como zonas que se intersectan generando dinámicas particulares.

\subsubsection{Actores/as}

La concurrencia en el territorio de actores/as provenientes de la sociedad civil para demandar la definición y concreción de políticas públicas (nacionales, provinciales y municipales) han configurado un arco de desempeños institucionales que no se limitan meramente al armazón estatal y han producido, por el contrario, lo que hemos dado a llamar "campos emergentes de interinstitucionalidad". Esa interinstitucionalidad que, en concreto está representada por diferentes actores/as sociales con pertenencia a disímiles disciplinas y trayectorias, constituye el ámbito en el cual, a nivel local, se gestan las políticas públicas. Para Stuchlik, "las relaciones interinstitucionales involucran una diversidad de actores, implican acciones conjuntas, toma de decisiones, enunciación e implementación de políticas, para lo cual se requieren procesos y espacios de coordinación. La perspectiva de entramado está relacionada con la

Revista Zona Franca- Centro de estudios interdisciplinario sobre las mujeres (CEIM)- Maestría poder y sociedad desde la problemática de género (MG), Rosario, Argentina. ISSN, 2545-6504 http://zonafranca.unr.edu.ar/index.php/ZonaFranca| Numero 27 (2019). 
existencia de espacios colegiados para la toma de decisiones, con participación de representantes de las instituciones corresponsables de las políticas" (Stuchlik. 2015: 54).

El abordaje de la violencia de género, requiere de la puesta en marcha de intervenciones interinstitucionales e interdisciplinarias dado que la problemática se presenta como multidimensional a partir de la propia constitución histórica. Esta complejidad -vinculada con dimensiones sociales, políticas, económicas, culturales, entre otras- no puede ser abordada en su compleción sino es mediante una estrategia de intervención interdisciplinaria en asociación de múltiples instituciones. Inclusive así también es explicitado en el denominado "Plan Nacional de Acción para la prevención, asistencia y erradicación de la violencia contra las mujeres 2017- 2019"'ll que buscó ejecutar el gobierno central: "el modelo de abordaje integral propuesto por este Plan Nacional de Acción incorpora los principios rectores de intersectorialidad e interinstitucionalidad. Es decir, el Consejo Nacional de las Mujeres es el órgano encargado del diseño de políticas públicas para prevenir, asistir y erradicar la violencia contra las mujeres en articulación y coordinación con los organismos gubernamentales con competencia en la materia y las organizaciones de la sociedad civil especializadas a nivel nacional, provincial y municipal. Para ello, el Plan Nacional propone un trabajo de fortalecimiento institucional como eje transversal a fin de afianzar y consolidar los mecanismos de coordinación y dar este modo una respuesta más eficaz ante las situaciones de violencia de género." (2016:20)

En relación con la intersectorialidad, hacemos mención a la forma de generar políticas públicas emanadas de acuerdos amplios entre diferentes instituciones provenientes de: la sociedad civil, el Estado y el sector privado. Tomando en consideración la particularidad local, los sectores que están compelidos a formar parte de la resolución de la problemática -y a los que hacemos referencia en este

III El Plan fue presentado en 2016 por el Consejo Nacional de las Mujeres. Organismo devenido luego en Instituto Nacional de las Mujeres (INAM) -creado por Decreto 968/2017 del Ejecutivo Nacional-, que pasó a funcionar bajo la órbita del Ministerio de Desarrollo Social.

Revista Zona Franca- Centro de estudios interdisciplinario sobre las mujeres (CEIM)- Maestría poder y sociedad desde la problemática de género (MG), Rosario, Argentina. ISSN, 2545-6504 http://zonafranca.unr.edu.ar/index.php/ZonaFranca| Numero 27 (2019). 
trabajo- son mayoritariamente estatales. Hay, sin embargo, una pequeña porción de representantes de instituciones de la sociedad civil con intereses particulares en la temática. En este sentido, Cunill-Grau sostiene que "cuando aludimos a la intersectorialidad en tanto relación entre distintos sectores gubernamentales, (...) la mirada aumenta su alcance y se coloca expresamente en la solución del problema que se busca enfrentar, advirtiendo que si el problema es multicausal, la integración sectorial puede mejorar su abordaje integral. Esto significa que es la búsqueda de la integralidad en el abordaje de un determinado problema o situación social, lo que coloca en el centro la cuestión de la relación entre distintos sectores gubernamentales" (2014: 02).

Si observamos cómo es y cómo ha sido en perspectiva la conformación de las partes interesadas que conforman esa interinstitucionalidad, podemos caracterizar someramente y agrupar a sus integrantes de la siguiente manera:

-Quienes inciden concreta y sistemáticamente a diario en el territorio. Están vinculadas a diversas instituciones del Estado (educación provincial, salud municipal, justicia provincial, dirección de políticas de género municipal y otras dependencias del municipio mayoritariamente), que, exceptuando algunos casos puntuales, carecen de formación específica en género.

-Académicas feministas que trabajan en diferentes proyectos de extensión/investigación en la Facultad de Ciencias Sociales - UNICEN. Que poseen formación en perspectiva de género y enfoque de derechos; muchas de ellas son activistas en espacios extra-académicos como el Frente Ni Una Menos Olavarría. Podríamos caracterizar sus intervenciones como cercanas a los posicionamientos del feminismo institucional (Varela, 2019).

-Personas que provienen de diferentes organizaciones de la sociedad civil o de asociaciones profesionales $u$ otros entes del Estado. Que como en el primer caso, no tienen formación en género exceptuando algunos casos puntuales (entre estos, las agrupaciones Animate, Se Puede, Espacio de contención y

Revista Zona Franca- Centro de estudios interdisciplinario sobre las mujeres (CEIM)- Maestría poder y sociedad desde la problemática de género (MG), Rosario, Argentina. ISSN, 2545-6504 http://zonafranca.unr.edu.ar/index.php/ZonaFranca| Numero 27 (2019). 
fortalecimiento para personas en situación de violencia de género. Y finalmente, todas aquellas agrupaciones que podríamos vincular con lo New Age).

Cabe mencionar que hay, también, por fuera de este repertorio, otras agrupaciones que no concurren a la Mesa y que sólo mencionaremos su existencia: Socorristas, Feministas Libertarias, Cara de Bicicleta (vinculada a la Juventud Guevarista), Plenario de Trabajadoras (PO) y Oleada Feminista. Son grupos de conformación reciente que, en general, descreen de las posibilidades que el Estado (en su estructura gubernamental actual) puede ofrecer para resolver problemáticas de violencia. En palabras de una integrante de Feministas Libertarias: “...quizás es que somos muy independientes del Estado por eso mismo no existe una relación ni siquiera buena o mala (...) Hablar del Estado también nos lleva a hablar de una cultura que legitima y que también es reproductora y generadora de todas las violencias. Si en algo estamos de acuerdo es que el Estado en la mayoría de los casos lo que hace es re victimizar a la víctima, violentarla desde sus instituciones como desde el hospital hasta la comisaría en caso que la víctima quiera hacer la denuncia. Así en muchos lugares. Las instituciones educativas tampoco están preparadas para un acompañamiento".

\subsubsection{Normativa}

De acuerdo con estándares internacionales, es obligación del Estado trabajar en la adopción de medidas integrales sobre quienes corren riesgos concretos de sufrir violencia de género para la salvaguarda de las personas. Nuestro país, respecto de esas obligaciones, ha sancionado en las últimas dos décadas una legislación acorde a esos estándares al tiempo que ha puesto en marcha -con altibajos- la normativa. En particular, resaltamos la Convención de Belém Do Pará, sancionada en 1994 en Brasil en el marco de la OEA. Documento que afirma que, por un lado, "la violencia contra la mujer constituye una violación de los derechos humanos y las libertades fundamentales y limita total o parcialmente a la mujer el

Revista Zona Franca- Centro de estudios interdisciplinario sobre las mujeres (CEIM)- Maestría poder y sociedad desde la problemática de género (MG), Rosario, Argentina. ISSN, 2545-6504 http://zonafranca.unr.edu.ar/index.php/ZonaFranca| Numero 27 (2019). 
reconocimiento, goce y ejercicio de tales derechos y libertades" y por otro, que los estados deben condenar "todas las formas de violencia contra la mujer y convienen en adoptar, por todos los medios apropiados y sin dilaciones, políticas orientadas a prevenir, sancionar y erradicar dicha violencia".

Todas las dimensiones del Estado han trabajado en la sanción de reglas que permitan propender a la igualdad -al menos en términos formales- para adecuarse a las obligaciones adquiridas desde el retorno democrático hasta el momento. Este contexto propició el advenimiento, a nuestras ciudades medias del centro bonaerense, de normativas para trabajar en consecuencia.

En referencia a la intersectorialidad, también observamos que es una categoría presente en una serie de documentos emitidos por dependencias del Poder Ejecutivo de la provincia de Buenos Aires ${ }^{\mathrm{IV}}$. En el año 2007 -en cumplimiento de la Ley provincial $\mathrm{N}^{\circ} 12569$ - se creó el Sistema Integrado Provincial para la Prevención y Atención de la Violencia Familiar (SIP), que entiende a la violencia familiar como una violación a los derechos humanos, como un problema político, social y de salud pública. En este sentido, se sostiene la necesidad de poner a disposición respuestas y recursos públicos, porque la problemática obstaculiza la construcción de relaciones democráticas al interior de las familias y la sociedad. Es por eso que el SIP se propuso generar políticas integrales, articuladas interinstitucionalmente y fortalecidas por una red de instituciones involucradas en la problemática.

El Ejecutivo bonaerense planteó el diseño del SIP como un espacio de confluencia de diversas instituciones, organismos públicos provinciales y locales, y organizaciones de la sociedad civil con trayectoria en el abordaje de la temática y la elaboración de acuerdos con los gobiernos municipales y el provincial. Para el

\footnotetext{
IV Documentos redactados en consonancia con la Ley Provincial de Violencia Familiar $N^{\circ} 12569$, el Decreto Reglamentario № 2785/06 y la Resolución MDH № 835/04: Sistema Integrado Provincial para la Prevención y Atención de la Violencia Familiar; Programa Provincial de Violencia Familiar. Mesas Locales; Protocolo del Rol del Acompañante; Protocolo de Admisión a los Servicios de Hospedaje Temporario.
}

Revista Zona Franca- Centro de estudios interdisciplinario sobre las mujeres (CEIM)- Maestría poder y sociedad desde la problemática de género (MG), Rosario, Argentina. ISSN, 2545-6504 http://zonafranca.unr.edu.ar/index.php/ZonaFranca| Numero 27 (2019). 
sostenimiento de estos andamiajes se impulsó la construcción de la figura de la "Mesa Local de prevención y atención de la violencia familiar" $\mathrm{V}$ integrada por áreas del gobierno municipal, organismos provinciales en territorio y grupos sociales y comunitarios. Cada Mesa Local apoyada en la Mesa Intersectorial Provincial, que está integrada por el Consejo Provincial de las Mujeres, la Secretaría de Derechos Humanos, el Ministerio de Desarrollo Humano, el Ministerio de Seguridad, el Ministerio de Salud, la Dirección General de Cultura y Educación, el Ministerio de Justicia y la Procuración de la Corte.

\subsection{La política pública local. Los dispositivos para atender la}

\section{violencia}

En Olavarría funciona desde el año 2010 la "Mesa Local contra la Violencia Familiar" con el objetivo de organizar, planificar y ejecutar intervenciones territoriales en relación con la problemática. En trabajos anteriores (Pérez, 2016; Pérez, 2018) profundizamos en torno de las actividades a lo largo de todo el período de trabajo en articulación con diferentes dependencias municipalesdesarrolladas en la ciudad: “(...) La Mesa Local tiene como propósito realizar tareas de prevención, asistencia y tratamiento a las víctimas de violencia, diseñando circuitos de intervención y delimitación de las competencias de cada una de las instituciones intervinientes" (2016: 256). Desde el 2011 la municipalidad gestiona y financia la puesta en marcha de un refugio para las personas víctimas de violencia, y la adquisición de botones antipánico -cuya distribución se realiza mediante la intervención del Juzgado de Familia $N^{\circ} 1$ de la ciudad y con monitoreo georreferenciado por parte del municipio desde 2012. También en 2011 se puso en práctica la Encuesta de Violencia Intrafamiliar desarrollada por el Observatorio de Seguridad Ciudadana para los Municipios de la Provincia de Buenos Aires; y en 2013 se pusieron en marcha grupos terapéuticos -tanto para personas en

\footnotetext{
${ }^{\vee}$ Nominación dada por Ley Provincial de Violencia Familiar № 12.569 Decreto Reglamentario № 2875/05 Resolución MDH № 835/04. En la práctica y en documentos posteriores, se reconoce a este espacio como "Mesa Local contra la violencia familiar y de género".
}

Revista Zona Franca- Centro de estudios interdisciplinario sobre las mujeres (CEIM)- Maestría poder y sociedad desde la problemática de género (MG), Rosario, Argentina. ISSN, 2545-6504 http://zonafranca.unr.edu.ar/index.php/ZonaFranca| Numero 27 (2019). 
situación de violencia como para sujetos violentos- desde la dependencia de Salud Mental municipal bajo la órbita de la Secretaría de Prevención y Atención Sanitaria. Desde el año 2014, la Mesa es parte de la denominada Red Preventiva de Asistencia Municipal ${ }^{\mathrm{V} !}$, integrada por distintas dependencias municipales y judiciales.

Todo ese andamiaje que mencionamos, se acompaña a nivel local con una ordenanza municipal $^{\mathrm{VII}}$ que proporciona presupuesto para el abordaje de las situaciones problemáticas analizadas.

La sanción de ese instrumento legal hizo que el gobierno local -bajo la administración de la alianza "Cambiemos"- produjera modificaciones en el organigrama del municipio desde diciembre de 2015, dando lugar a la creación de la DPG. La oficina municipal tiene como objetivo la atención de las personas que han atravesado o atraviesan violencia de género. Esta Dirección institucionaliza una gran parte de los reclamos sostenidos, de diferentes maneras, en la ciudad por algunas representantes del feminismo local. En este sentido, el surgimiento de la DPG puede inscribirse en el marco de esas demandas por un lado, pero también en cierto impulso -en lo discursivo sobre todo- que la nueva alianza gobernante decide otorgar a la problemática. ${ }^{\text {VIII }}$ La DPG olavarriense irrumpe en un contexto local donde era urgente trabajar institucionalmente dada la profusa notoriedad que tomó por ese tiempo la violencia extrema. Por un lado, la ciudad

VI La Red se conforma por la Secretaría de Desarrollo Social, la Secretaría de Prevención y Atención Sanitaria, la Unidad Coordinadora de Políticas Preventivas, el Hospital Municipal, el Hospital Municipal de Hinojo, el Servicio de Promoción y Protección de los Derechos del Niño, el Centro Preventivo de las Adicciones, la Comisaría de la Mujer y la Familia, el Programa de Prevención y Asistencia al Juego Compulsivo, la Casa del Adolescente, el Servicio de Nutrición del Hospital Municipal, la Dirección de Políticas de Integración en Discapacidad, el Centro de Día Municipal para la Atención a las Adicciones, la Defensoría Pública Civil y Comercial № 1, Servicio Zonal de Promoción y Protección de los Derechos del Niño, el Juzgado de Familia $\mathrm{N}^{\circ}$ 1, la Secretaría Especializada en Violencia Familiar y de Género del Ministerio Público.

${ }_{\text {VII }} N^{\circ} 3910 / 15$ de Atención Integral de la Violencia contra las mujeres, Género y Familiar.

VIII Durante la presidencia de Mauricio Macri (2015-2019) se puso en marcha a nivel nacional el "Plan Nacional de Acción para la prevención, asistencia y erradicación de la violencia contra las mujeres 2017-2019". Contemporáneamente, en la provincia de Buenos Aires -y bajo el mismo signo político que la nación- aparece el llamado "Plan Integral de Género" que se presentó como un instrumento para proteger a la mujer ante casos de violencia.

Revista Zona Franca- Centro de estudios interdisciplinario sobre las mujeres (CEIM)- Maestría poder y sociedad desde la problemática de género (MG), Rosario, Argentina. ISSN, 2545-6504 http://zonafranca.unr.edu.ar/index.php/ZonaFranca| Numero 27 (2019). 
estaba fuertemente conmovida por dos casos de femicidios cercanos en el tiempo y sobre los que se sucedieron una serie de acciones de reclamo para pedir justicia públicamente con manifestaciones callejeras y pedidos con incidencia en los medios locales por los crímenes ${ }^{\mathrm{IX}}$.

La nueva dependencia puso en marcha su dispositivo de abordaje y prevención a instancias de la sanción de esa nueva ordenanza que -como explicitamos- proporciona presupuesto para trabajar con situaciones problemáticas. De modo tal que, la nueva Dirección contó desde el inicio para su funcionamiento con un presupuesto que equivale al $0,3 \%$ del erario anual municipal. Sin embargo, a pesar de los recursos particulares que posee la DPG no sucede lo mismo con otras áreas clave del municipio ${ }^{X}$; no es menor señalar el contexto adverso en términos económicos y políticos que se corresponde con las lógicas neoliberales implementadas desde la asunción al gobierno de la alianza Cambiemos. La degradación engloba también otros planos de la vida. En sus escasos años de existencia, la DPG ha llevado adelante una innumerable cantidad de acciones que se enmarcan en dos grandes áreas: una de promoción, prevención y sensibilización -compuesta por comunicadoras sociales mayoritariamente-, y la otra de intervención en situaciones de violencia -integrada por trabajadoras sociales, psicólogas, acompañantes terapéuticas y abogadas. Durante el período de análisis (2015-2018) atendieron alrededor de 800 situaciones (cada una de las mismas puede requerir más de una intervención según la necesidad de que se trate). Recientemente, desde mediados de 2018, están incursionando en un espacio socioterapéutico en torno de la reflexión sobre la construcción social de las masculinidades violentas dado el convencimiento que la gestión de la DPG tiene en torno de trabajar con los hombres que ejercen violencia a los efectos de romper el circuito patriarcal que la posibilita.

\footnotetext{
IX Se trata de los femicidios de Tamara Bravo y Graciela Tirador.

$x$ Por ejemplo, hacemos referencia a otras áreas de la misma Secretaría de Derechos, Igualdad y oportunidades como lo son: la Dirección del Servicio Local de Promoción y Protección de los Derechos del Niño; la Dirección de políticas de integración para la discapacidad y la Dirección de niñez, adolescencia y adicciones.
}

Revista Zona Franca- Centro de estudios interdisciplinario sobre las mujeres (CEIM)- Maestría poder y sociedad desde la problemática de género (MG), Rosario, Argentina. ISSN, 2545-6504 http://zonafranca.unr.edu.ar/index.php/ZonaFranca| Numero 27 (2019). 
A partir de esta nueva conformación institucional, la DPG ha desarrollado una estrecha vinculación con la Mesa local contra la Violencia Familiar. En este sentido, focalizamos la mirada en torno de las tensiones aparecidas en la interacción institucional a partir de la reinstauración de un contexto sociopolítico en el cual se evidencia con claridad el achicamiento del Estado vinculado a cuestiones de índole social ${ }^{\mathrm{X}}$. Así, indagamos entonces en dos planos; por un lado, cómo se reordena el territorio -entendido como un espacio social atravesado por tramas complejas y en constante tensión- y por otro, cómo el nuevo dispositivo estatal ha logrado dar respuesta escasa al tema.

\section{Sobre la interacción interinstitucional}

Nos interesa poner de manifiesto -considerando el contexto sociopolítico ${ }^{\mathrm{XII}}$ las tensiones que surgen en la interacción institucional; así, intentaremos ver cómo se ha reordenado el territorio -pensado como un espacio social atravesado por tramas complejas y en constante tensión- en apenas unos pocos años. El reordenamiento al que aludimos, sucedido en la ciudad media estudiada, tiene que considerar necesariamente la aparición en la escena política local de la DPG.

La relación institucional que ha tenido lugar entre la Mesa y la DPG experimentó diversos momentos; a continuación, nos referiremos a estos como primera y segunda etapa.

\footnotetext{
${ }^{\mathrm{X}}$ No abordaremos en este espacio nociones vinculadas con las ideas de neoliberalismo dado que su incorporación derivaría en otras discusiones que nos alejaría del tema.

XII De acuerdo a datos del Observatorio de Derechos Humanos de la Ciudad de Buenos Aires, tomando como referencia 2015, en 2016 se destinaron 50,38\% menos recursos al Programa Nacional de Educación Sexual Integral (ESI); y en 2017 un 22,5\% por debajo. En 2015 se logró la reglamentación del artículo 11 de la Ley de Identidad de Género -que garantiza a las personas trans el acceso a la salud integral- sin embargo, no se observan avances en la apertura de consultorios amigables para la diversidad sexual. El proyecto de presupuesto 2018 destinaba al Instituto Nacional de las Mujeres (ex Consejo Nacional de las Mujeres) al menos 2 millones de pesos menos que el año anterior y además no contemplaba la inflación estimada oficialmente. Finalmente, y a instancias de un pedido efectuado por diferentes organizaciones (ELA, CAREF, MEl y Fundación Siglo 21), en enero de 2018 se destinó un monto extra de 50 millones de pesos. La reducción presupuestaria inicial, propuesta por el oficialismo, se traducía en menos recursos disponibles para la aplicación del Plan Nacional de Acción para la Prevención. Asistencia y Erradicación de la Violencia contra las Mujeres 2017-2019 (Pérez e Iturralde, 2018).
}

Revista Zona Franca- Centro de estudios interdisciplinario sobre las mujeres (CEIM)- Maestría poder y sociedad desde la problemática de género (MG), Rosario, Argentina. ISSN, 2545-6504 http://zonafranca.unr.edu.ar/index.php/ZonaFrancal Numero 27 (2019). 


\subsection{Primera etapa: reacomodamiento del territorio.}

Podríamos denominar a esta primera etapa, como de reacomodamiento territorial. Dado que al tiempo que se conformaba la estructura municipal de funcionamiento de la DPG, se produjeron ciertos resquemores entre las/os actores de mayor trayectoria de la Mesa. Dado que la institucionalidad emergente no terminaba de acomodar su misión social en la complejidad del territorio y en la organización -también compleja- de la abrumadora tarea que encaraba respecto de conjurar la violencia.

En un principio, la DPG fue la depositaria de las expectativas de resolución de la conflictiva de género para varios actores sociales locales, sobre todo para quienes estaban militando causas feministas desde antes. Incluso, el intendente local ${ }^{\mathrm{XIII}}$-mentor de la iniciativa- afirmó que: "surgió la necesidad de tener un lugar de contención para las víctimas de violencia de género. Es clave tener un espacio de confidencialidad y trabajar tranquilos con las víctimas" (Infoolavarría, 2016). También, observamos cómo desde la Mesa -que antecede en tiempo y acciones a la novel dependencia-, se producen una serie de reacciones al incorporarse a las reuniones mensuales.

En el inicio, más allá de la endeblez en términos del manejo político y en torno del conocimiento del tema de sus primeras titulares -que necesitaban una mayor formación en género y mejor capacidad para el manejo de grupos de trabajo-, se da una fuerte disputa sobre los usos que debían hacerse del presupuesto asignado por la Ordenanza No 3910/15 a la DPG. Parte de la Mesa sostuvo la necesidad de incorporar al $0,3 \%$ asignado los montos que ya estaban siendo utilizados a los efectos de solventar las acciones realizadas por la misma acorde a lo que establece el Artículo 4 de la ordenanza ${ }^{\mathrm{XIV}}$ (entre estos, la compra

\footnotetext{
XIII Se trata de Ezequiel Galli de la alianza Cambiemos

XIV Art. $4^{\circ}$ : La partida presupuestaria creada por el Art. $1^{\circ}$, "sin perjuicio de otros recursos asignados por cualquier otra área de jurisdicción Municipal, Provincial y/o Nacional, se destinará a promover, garantizar y/o profundizar políticas que contemplen la perspectiva de género, con el
}

Revista Zona Franca- Centro de estudios interdisciplinario sobre las mujeres (CEIM)- Maestría poder y sociedad desde la problemática de género (MG), Rosario, Argentina. ISSN, 2545-6504 http://zonafranca.unr.edu.ar/index.php/ZonaFranca| Numero 27 (2019). 
de botones antipánico, la impresión de alguna folletería. Incluía también la realización de alguna jornada de capacitación). Sin embargo, la evidencia de la pérdida económica no fue considerada como tal para la titular de ese momento: "yo voy a hablar de lo técnico, no de lo político" y además recalcó que: "Hay decisiones políticas que se tienen que tomar... cada una puede hacer el reclamo como lo considere, como Dirección entendemos como lo explican" (en referencia a cómo se argumentaba con tecnicismos contables la pérdida de un porcentaje de presupuesto por parte de administrativos del gobierno local) ${ }^{\mathrm{XV}}$. Otros actores sostuvieron que no había razón de queja: "si ahora tenemos 2.700.000 de qué nos estamos quejando"XVI. En este aspecto, cabe agregar que el cruce se dio al interior de una de las reuniones mensuales de Mesa, en la que se marcaron fuertemente las diferencias entre las variadas vinculaciones, simpatías o acercamiento político partidario de sus integrantes: quienes estaban ideológicamente consustanciados con la alianza gobernante Cambiemos y quienes estaban en la oposición política. Una de las actoras clave de la Mesa expresó: "la Mesa es esa articulación, el tema es que por supuesto tiene todos los atravesamientos... Nosotros hemos estado atravesados por... situaciones de mucho atravesamiento político de querer capitalizar políticamente esto para algún sector... eso por suerte se pudo ir mediando, manejando... Y eso es lo que le da la autonomía hoy... que... digamos yo creo que de los organismos que existieron, de las inter organizaciones interinstitucionales que existieron desde la década anterior es lo único que hemos sobrevivido...". Pudimos verificar, en este sentido, algunas situaciones de malestar vinculadas a la "aparición de dinero". Entre los reclamos más sentidos, se solicitó que se clarifiquen las erogaciones de la DPG a los efectos de poder planificar acciones en el territorio y saber qué montos se

objetivo de erradicar de manera progresiva todo tipo de violencia contra las mujeres en el Partido de Olavarría".

XV Para el año 2016, de acuerdo a la ordenanza 3910/15, a la Dirección de Políticas de Género le correspondía administrar un total de 2.700 .000 pesos. La gestión "Cambiemos" pensaba incluir en la partida los $\$ 300.000$ que ya se venían invirtiendo como resultado de acciones desarrolladas por la Mesa. Esto significaba contar con un $11 \%$ menos de presupuesto.

XVI Abogado que en ese momento participaba de la Mesa representando "Olavarría Despierta", organización de la sociedad civil dedicada a trabajar con temáticas de abusos infantiles.

Revista Zona Franca- Centro de estudios interdisciplinario sobre las mujeres (CEIM)- Maestría poder y sociedad desde la problemática de género (MG), Rosario, Argentina. ISSN, 2545-6504 http://zonafranca.unr.edu.ar/index.php/ZonaFrancal Numero 27 (2019). 
habían destinado a qué aspectos (refugio y mantenimiento del mismo, subsidios a personas en situación de vulnerabilidad, proyectos). La presentación de los datos de acuerdo a la construcción del presupuesto municipal en un sistema informático denominado RAFAM (Reforma de la Administración Financiera en el Ámbito Municipal) hizo que la lectura de los mismos sea ininteligible. Otra integrante de la mesa sostuvo en uno de los plenarios mensuales: "estos programas que no entendemos nada están hechos a propósito para que no entendamos y para cubrirse ellos... ¿cómo vamos a hacer para saber en qué se gastan la plata de la ordenanza? porque si dicen que los datos están ahí... ponele que están, pero como no los entendemos nos pueden joder como quieren".

Un ejemplo de este escaso manejo institucional para adecuarse a la lógica tensionante del juego político, lo muestra el lanzamiento, desde la DPG, del segundo llamado para presentación de proyectos destinados a cubrir -con acciones de capacitación o sensibilización-, aquellos espacios que la propia dependencia no podía abarcar. Como resultado, se presentaron 17 propuestas de variada índole. Muchas de estas, muy reñidas con los supuestos métodos de intervención que enarbola la racionalidad estatal; en este sentido, aparecieron algunas ideas a las que, si bien se les dio curso por decisión política, no venían a solucionar ningún aspecto de las necesidades más sentidas de las mujeres violentadas. Las propuestas a las que aludimos están vinculadas al "new age" (Carozzi, 2002) como la biodanza, la bioenergética y otras prácticas similares; todas carecían de perspectiva de género e incluso algunas apelaban a ciertas ideas esenciales en torno del hecho de ser mujer. Queda en claro que no se desestima el -tal vez posible- potencial de estas actividades para algunos ámbitos y sujetos. Sin embargo, a criterio posterior de la DPG, no constituyen herramientas para utilizar en grupos vulnerables como los son las mujeres en situación de violencia donde las necesidades materiales concretas y percibidas como tales que son vivienda y trabajo- no se encuentran satisfechas ni resueltas ${ }^{\mathrm{XVII}}$. Otras

XVII Uno de los proyectos expresa: "Se proponen encuentros culturales femeninos con herramientas corporales y terapéuticas (yoga, biodanza, bioenergética, expresión corporal, círculo de cultura,

Revista Zona Franca- Centro de estudios interdisciplinario sobre las mujeres (CEIM)- Maestría poder y sociedad desde la problemática de género (MG), Rosario, Argentina. ISSN, 2545-6504 http://zonafranca.unr.edu.ar/index.php/ZonaFranca| Numero 27 (2019). 
presentaciones -ante la no regulación de los aspectos del llamado para la presentación de propuestas- pasaron presupuestos muy onerosos; en otros casos omitieron hacerlo, apelando al voluntarismo. Otros grupos, en cambio, proponían trabajar con aspectos que, si bien eran colindantes con acciones de sensibilización o acompañamiento con poblaciones vulnerables, no contribuían estrictamente con los propósitos de la DPG (por ejemplo, el abuso sexual infantil). Finalmente, algunas propuestas se solaparon con las intervenciones que la misma DPG efectúa. La variedad de propuestas para su ejecución -con estipendio en la mayoría de los casos-, se puede vincular con la decisión tomada en el primer "llamado a propuestas", que ante la carencia de indicaciones sobre qué aspectos resultaban prioritarios para complementar la tarea de la DPG, la decisión política del momento fue la de aprobar todas las presentaciones.

\subsection{La segunda etapa: mayor racionalidad y academicismo}

Esta segunda etapa, que tampoco está exenta de tensiones, podemos vincularla con cierta idea de consolidación institucional; es coincidente con el momento en el cual la gestión de la DPG cambió a manos de su nueva titular (efectuada en junio de 2016); allí se conforma un equipo de trabajo más amplio integrado por diferentes profesionales con perfiles y/o formación más idóneos para trabajar con mujeres violentadas. Hubo, entonces, un incremento significativo de profesionales y una agenda política que marcó la necesidad de relacionamiento con otros espacios neurálgicos del municipio (salud, desarrollo social, Servicio Local de Promoción y Protección de los Derechos del Niño) como así también con las otras instituciones de la provincia. Sobre todo, se ahondaron lazos institucionales con el sistema judicial y las instancias encargadas de trabajar con violencia de género y diversidad en esa dimensión estatal.

trabajo de Programación Neurolingüística), herramientas lúdicas (teatro, música y juegos) y herramientas de sustentabilidad económica (tejido, costura, trabajo con la tierra)." Otro proponía la "gestión emocional para la transformación social a través de la Biodanza". (Fuente: Dirección de Políticas de Género. Agosto de 2018).

Revista Zona Franca- Centro de estudios interdisciplinario sobre las mujeres (CEIM)- Maestría poder y sociedad desde la problemática de género (MG), Rosario, Argentina. ISSN, 2545-6504 http://zonafranca.unr.edu.ar/index.php/ZonaFranca| Numero 27 (2019). 
En relación a las tensiones aparecidas en la primera etapa en torno del presupuesto que maneja la DPG, en este período se determinó solicitar informes semestrales al Ejecutivo para que responda en torno de las erogaciones realizadas, y así cubrir la demanda de información que requieren las integrantes de la Mesa a los efectos de sugerir acciones a la Dirección.

En vinculación a la presentación de propuestas para coadyuvar a las tareas de la DPG, a los efectos de orientar las presentaciones y para evitar las situaciones dadas con anterioridad en los llamados previos, la dependencia creó "áreas de vacancia" con correspondencia -básicamente- en dos tipos de situaciones: primero, las necesidades sentidas por las mujeres sobre la que se producen las intervenciones; y segundo, aquellas consideradas como necesarias para un mejor conocimiento en torno del funcionamiento de la denominada "ruta crítica" (Sagot Rodríguez y Carcedo, 2000) y para producir intervenciones concretas en espacios como el de la salud municipal. Asimismo, aquellas propuestas consideradas "viables" por la DPG, comenzaron a ser evaluadas por una terna de juradas, compuesta por académicas provenientes de ciudades medias de la región centro, conocidas todas por su trayectoria en temáticas afines. Estas decisiones, que son más congruentes con la lógica vinculada a la racionalización de las actividades del Estado, provocaron también rispideces con actoras sociales otrora favorecidas con subsidios a propuestas de corte alternativo. Particularmente, en el seno de la Mesa, entre quienes enarbolaban la defensa de las posturas de resolución de problemas de violencia de género con propuestas alternativas, se argumentó la disconformidad de la selección resultante, dado que los proyectos escogidos tenían un fuerte componente y matiz académico/academicista. Hubo voces que objetaron la predominancia de las propuestas académicas: "todos los proyectos que ganaron son de la Facultad".

Finalmente, una vez consolidado el equipo de trabajo de la DPG, se hizo evidente cierta tensión entre quienes se desempeñan como profesionales de la dependencia, específicamente en el área de abordaje y el resto de los/as

Revista Zona Franca- Centro de estudios interdisciplinario sobre las mujeres (CEIM)- Maestría poder y sociedad desde la problemática de género (MG), Rosario, Argentina. ISSN, 2545-6504 http://zonafranca.unr.edu.ar/index.php/ZonaFranca| Numero 27 (2019). 
integrantes de la Mesa; en algunas oportunidades, las profesionales de la Dirección objetan los reclamos/solicitudes provenientes del resto de las personas que la integran. Para ellas, existe un cierto desconocimiento respecto del trabajo cotidiano y propio de la DPG, del territorio y del tratamiento de temas de altísima conflictividad. En este sentido, se esgrime que los casos de violencia vienen aparejados con vulneraciones de derechos, trayectorias escolares inconclusas, a veces están vinculados con consumos problemáticos de sustancias, con necesidades materiales históricas y actuales no resueltas, con el ejercicio de la prostitución, con cuidados "problemáticos" con niños/as, con imposibilidades de comprensión del peligro vital que implica la propia violencia de género, con descreimiento de la capacidad de intervención del Estado en el caso particular y porque se desconoce el entramado formal e informal de intervenciones interinstitucionales que se realiza en cada situación que se presenta. Al respecto: "Viste... nosotras hacemos mucho, hacemos lo posible y lo imposible a veces, tocamos a las trabajadoras sociales de los territoriales que conocemos para ver qué pasa con esa mujer o con esa familia, porque tienen datos seguros; otros días no tenés descanso, pero nada alcanza. Porque no entienden lo que nosotras hacemos, no entienden lo que hay que remar acá adentro, del Estado digo, no acá en la Dire, para que te den un poco de bola con las necesidades de la gente. $Y$ bueno, nada alcanza, nada alcanza". La entrevistada menciona la utilización de canales informales para intentar abordar y resolver algunas de las situaciones que se atienden en la dependencia, que van desde el uso del propio teléfono hasta la solicitud de aceleración de trámites vinculados con las mujeres atendidas a conocidos/as de otras dependencias del Estado.

En esta segunda etapa -respecto de la Mesa- puede evidenciarse una altísima rotación de las personas que representan diferentes instituciones concurrentes a la misma; esto hace que, desde el uso de un lenguaje en común, hasta la dinámica de funcionamiento y el consenso necesario para la toma de decisiones, en algunas oportunidades, se ralentice (dada esa lógica de

Revista Zona Franca- Centro de estudios interdisciplinario sobre las mujeres (CEIM)- Maestría poder y sociedad desde la problemática de género (MG), Rosario, Argentina. ISSN, 2545-6504 http://zonafranca.unr.edu.ar/index.php/ZonaFranca| Numero 27 (2019). 
concurrencia no sistemática). En este sentido, para algunas actoras claves de la Mesa, habría un eterno retorno al punto de inicio fundacional cada vez que se incorpora alguna persona nueva en representación institucional. Entre otras cuestiones, se argumenta que se desconoce la trayectoria de la Mesa, así como también las acciones que han efectuado y las posibilidades concretas que tienen de intervención específica en el marco de la denominada "ruta crítica".

También se evidencia en esta segunda etapa una merma de integrantes de organizaciones de la sociedad civil: este es el caso del Frente Ni Una Menos, que está presente desde 2015 en la ciudad y aunque sigue activo se vincula esporádicamente para la realización de acciones concretas con la DPG, más no con la Mesa. Sucedió lo mismo con otros grupos más pequeños, con escasa solidez institucional vinculados a las prácticas y terapias alternativas; en estos casos en particular, visualizamos que a posteriori de la desaprobación de las propuestas presentadas, dejaron de participar de las reuniones mensuales.

\section{Conclusiones provisorias}

Si tomamos en consideración la particularidad local, los sectores que están compelidos a formar parte de la resolución de la problemática de violencia de género, y a los que hacemos referencia en este trabajo, son mayoritariamente estatales.

Provisoriamente, podemos afirmar que se evidencia la necesidad de seguir construyendo reflexiones, debates y diálogo consensuado e interdisciplinario al interior de esta formación institucional como lo es la Mesa, que condensa en su constitución a casi todos los organismos estatales que pueden prevenir o abordar situaciones de violencia. Asimismo, se percibe como necesario la construcción de una estrategia comunicacional que pueda transmitir necesidades, inquietudes, dudas, posicionamientos de cada una de las instituciones participantes hacia el interior de la propia Mesa, como así también para el afuera. Si de algo carecen

Revista Zona Franca- Centro de estudios interdisciplinario sobre las mujeres (CEIM)- Maestría poder y sociedad desde la problemática de género (MG), Rosario, Argentina. ISSN, 2545-6504 http://zonafranca.unr.edu.ar/index.php/ZonaFranca| Numero 27 (2019). 
tanto la Mesa como la DPG es de una estrategia comunicacional adecuada. Parafraseando a Washington Uranga: "¿Se puede hacer todo esto sin comunicación? Definitivamente no. La gestión de políticas públicas requiere de la comunicación en términos integrales. Como proceso de intercambio y diálogo entre los actores que conforman la escena pública y como necesario instrumento de difusión de información. También como estrategia para la implementación. Es impensable desarrollar políticas públicas sin acudir al aporte de la comunicación." (Uranga, 2012: 6)

Territorio, actores sociales e institucionales, dispositivos de abordaje y presupuestos locales, mayor demanda de atención de situaciones dado el incremento "de la agencia por los derechos de las mujeres" y la menor tolerancia hacia la violencia (Barrancos: 2018) y contexto, han cambiado cuantiosamente en un breve lapso de tiempo. Sin embargo, la violencia sigue presente en la ciudad media mientras se disputan sentidos en torno de intereses institucionales particulares que aparecen como generales.

En este sentido, que se pueda comprender cuáles son las funciones y competencias particulares de cada una de las partes intervinientes (tanto de las instancias del Estado como del resto de las organizaciones de la sociedad civil) en la prevención y tratamiento de la temática, sería una buena estrategia a los efectos de reafirmar la validez de la participación en la Mesa. También, sería necesario que se entable la necesidad de "construir información permanente sobre los temas" (Uranga, 2012) y recursos presentes y necesarios en territorio que se consideran de relevancia para poner en agenda pública y política, a los efectos de tender hacia la erradicación -consensuada en acciones y discursos- de la violencia machista. Si bien las instituciones integrantes de la Mesa y propia DPG no han podido conjurar la violencia machista - y estimamos que para su erradicación hará falta el derrumbe de lo establecido al tiempo de pensar la construcción de una contracultura que pueda incorporar fundacionalmente la importancia por el respeto a los derechos humanos-, al menos se ha logrado su establecimiento y

Revista Zona Franca- Centro de estudios interdisciplinario sobre las mujeres (CEIM)- Maestría poder y sociedad desde la problemática de género (MG), Rosario, Argentina. ISSN, 2545-6504 http://zonafranca.unr.edu.ar/index.php/ZonaFranca| Numero 27 (2019). 
reconocimiento ciudadano, hecho que se corrobora en la demanda de atención sostenida en casos de violencia de género.

Particularmente, sostenemos que si bien el Estado no es la institución que idealmente pudiera modificar el orden de cosas dado y con capacidad para resolver para siempre las situaciones problemáticas (de violencia y de cualquier tipo) tampoco puede obviarse su contundente presencia en tanto institución que ha marcado a las sociedades desde su surgimiento. Menos puede desconocerse su potencial como posibilitador de estructura, profesionales y recursos para modificar situaciones de vulnerabilidad. El Estado es un actor privilegiado de la política pública, porque "interviene como estructura burocrática y representación simbólica dentro del campo de fuerzas donde se dirimen cuáles son los temas y los modos para su resolución" (Oszlak y O'Donnell: 1995). Si bien es una institución de raigambre patriarcal, preferimos aquellas posturas que sostienen la necesidad de filtrarnos en sus intersticios a los efectos de poder modificarlo, incidiendo desde adentro tanto en su propia estructura como en la sociedad toda.

\section{Bibliografía}

BARRANCOS, D. (2018) Violencia Patriarcal. Jornadas de Género en Paraná: capacitación, debate y reflexión sobre la igualdad y equidad de género. Recuperado de www.bomberosra.org.ar/4-encuentro-genero/disertaciones/1.pdf

BARRERA BASSOLS, Dalia y MASSOLO, Alejandra (1998) Mujeres que gobiernan municipios. Experiencias, aportes y retos. PIEM/COLMEX, México.

BOGGI, Silvia. (2008) Transformaciones, incertidumbres y capturas efímeras: reconversión de imaginarios urbanos de ciudades bonaerenses de rango medio. Newsletter, 13. Olavarría: FACSO - UNICEN.

BOGGI, Silvia y GALVÁN, Nora (2016). Ciudad media, ciudad intermedia ¿ni chicha ni limonada? En: Gravano, A.; Silva, A. y Boggi, S. (Eds.) Ciudades vividas: 
sistemas e imaginarios de ciudades medias bonaerenses. Buenos Aires: Café de las Ciudades.

CAROZZI, María Julia (2002) Definiciones de la New Age desde las Ciencias Sociales. Boletín de Lecturas Sociales y Económicas, 5 (2). Buenos Aires: UCAFCSE. Recuperado de http://200.16.86.50/Digital/33/revistas/blse/carozzi1-1.pdf

CONSEJO NACIONAL DE LAS MUJERES (2016) Plan Nacional de Acción para la Prevención, Asistencia y Erradicación de la Violencia contra las mujeres 2017-2019. Ministerio de Salud y Desarrollo Social. Recuperado de https://www.argentina.gob.ar/inam/plandeaccion

CUNILL-GRAU, Nuria (2014). La intersectorialidad en las nuevas políticas sociales: Un acercamiento analítico-conceptual. Gestión y política pública, 23 (1), 5-46.

Recuperado de

http://www.scielo.org.mx/scielo.php?script=sci arttext\&pid=S14051079201400010 0001\&lng=es\&tlng=es.

HARAWAY, Donna (1995) Ciencia, cyborgs y mujeres. La reinvención de la naturaleza. Madrid: Ediciones Cátedra.

INFOOLAVARRIA (8 de agosto de 2016) Vidal inauguró formalmente la sede de la Dirección de Políticas de Género. Recuperado de https://infoolavarria.com/2016/08/08/vidal-inauguro-formalmente-la-sede-de-la direccion-de-politicas-de-genero/

ITURRALDE, María Eugenia (2017). De medios e intermedias: medios de comunicación en ciudades intermedias. lluminuras, 18 (45). Recuperado de https://seer.ufrgs.br/iluminuras/article/view/79128/45964

INSTITUTO NACIONAL DE ESTADÍSTICAS Y CENSOS (INDEC) (2010) Censo Nacional de Población, Hogares y Viviendas 2010. Recuperado de https://www.indec.gob.ar/nivel4 default.asp?id tema $1=2 \&$ id tema $2=41$ \&id tem a $3=135$

LASSWELL, Harold D. (2013) La concepción emergente de las ciencias de políticas. En Aguilar Villanueva, Luis (Ed.) El estudio de las Políticas Públicas. México: MAPorrúa.

Revista Zona Franca- Centro de estudios interdisciplinario sobre las mujeres (CEIM)- Maestría poder y sociedad desde la problemática de género (MG), Rosario, Argentina. ISSN, 2545-6504 http://zonafranca.unr.edu.ar/index.php/ZonaFranca| Numero 27 (2019). 
OSZLAK, Oscar, y O'DONNELL, Guillermo (1995). Estado y políticas estatales en América Latina: hacia una estrategia de investigación. Redes, 2 (4), 99 - 128. Quilmes, UNQUI.

PÉREZ, Patricia (2016). Construcción de una agenda política con perspectiva de género en la ciudad media. En A. Gravano , A. Silva, y S. Boggi, Ciudades vividas. Sistemas e imaginarios de ciudades medias bonaerenses, 373 388. CABA, Argentina: Café de las Ciudades.

PÉREZ, Patricia (2018) Hacia un primer análisis en torno de una institución local que aborda problemáticas de violencia de género. ENACOM - FADECCOS. Olavarría. En prensa.

PÉREZ, Patricia e ITURRALDE, María E. (2018) Género y agendas en una ciudad intermedia. Question. 1 (58). Recuperado de http://perio.unlp.edu.ar/ojs/index.php/question/

SAGOT RODRíGUEZ, Monserrat y CARCEDO, Ana (2000) Ruta crítica de las mujeres afectadas por la violencia intrafamiliar en América Latina. Estudios de caso en diez países. Washington DC: Organización Panamericana de la Salud Programa Mujer, Salud y Desarrollo. Recuperado de http://ns.bvs.hn/docum/ops/libros/rutacritica.pdf

STUCHLICK, Silvia (2015) Capacidades estatales para la gobernabilidad de los sistemas de protección de derechos. Estudio de caso: El sistema de protección integral de Derechos de Niños, Niñas y Adolescentes de la ciudad de Buenos Aires: 1996-2012. Tesis de Maestría en Administración y Políticas Públicas. Buenos Aires: UDESA.

URANGA, Washington (2012) Sin comunicación democrática no hay políticas públicas democráticas. En RINALDI, L. (coord.) Perspectivas y experiencias de comunicación política. Buenos Aires: La Crujía.

VARELA, Nuria (2019) Feminismo para principiantes. Ciudad Autónoma de Buenos Aires: B de Books.

Revista Zona Franca- Centro de estudios interdisciplinario sobre las mujeres (CEIM)- Maestría poder y sociedad desde la problemática de género (MG), Rosario, Argentina. ISSN, 2545-6504 http://zonafranca.unr.edu.ar/index.php/ZonaFranca| Numero 27 (2019). 